\section{AL-AZHAR Dental Journal}

F $\quad \mathrm{o} \quad \mathrm{r}$
$\mathrm{G}$
The Official Publication

of The Faculty of Dental

Medicine For Girls,

Al-Azhar University Cairo, Egypt.

Print ISSN 2537-0308 • Online ISSN 2537-0316

ADJ-for Girls, Vol. 8, No. 4, October (2021) - PP. 577:582

\title{
Effect of Rapid Prototyped Upper Complete Denture on Retention and Patient Satisfaction
}

\author{
Nahla A. Zohny ${ }^{1 *}$, Mostafa M. Abdel-Ghany ${ }^{2}$, Asmaa A. Ashour ${ }^{3}$
}

Codex : 06/21.10

azhardentj@azhar.edu.eg

http://adjg.journals.ekb.eg

DOI: $10.21608 /$ adjg.2021.41596.1289

Restorative Dentistry

(Removable Prosthodontics, Fixed

Prosthodontics, Endodontics, Dental Biomaterials, Operative Dentistry)

\section{KEYWORDS}

CAD/CAM dentures,

Rapid prototyping, Retention,

Satisfaction.

\begin{abstract}
Purpose: This study aimed to evaluate the effect of rapid prototyped upper complete denture on retention and patient satisfaction. Material and methods: Eight completely edentulous patients were selected for this study with ages ranged from 40-50 years. All patients had received two complete dentures one fabricated by conventional heat compressed method and the other fabricated by rapid prototyping technology. Patients were classified into two groups, four patients each. Group I received the conventional denture first then the rapid prototyped denture while, Group II received the rapid prototyped denture first then the conventional denture. All patients were instructed to wear their dentures for equal periods of 1 month where retention and patient satisfaction were measured at insertion and after 1 month. After an interval of two weeks, as a wash out period, patients received their other denture. Results: The results of this study showed that there was a high statistical significant difference between the conventional and the rapid prototyped denture regarding retention and patient satisfaction while there was no significant difference between both groups, group I which received the conventional denture first and group II which received the rapid prototyped denture first. Conclusion: within the limitation of this study, it was concluded that retention and patient satisfaction of maxillary rapid prototyped denture were significantly higher than that of the conventional denture. The rapid prototyped dentures can be used as an alternative effective satisfactory treatment for completely edentulous patients rather than conventional dentures but furthermore studies are needed.
\end{abstract}

- Paper extracted from Master thesis tilted "Effect of Rapid Prototyped Upper Complete Denture on Retention and Patient Satisfaction"

1. Dentist, Ministry of Health, Egypt

2. Professor of Removable Prosthodontics, Faculty of Dental Medicine for Girls, Al-Azhar University, Cairo, Egypt.

3. Lecturer of Removable Prosthodontics, Faculty of Dental Medicine for Girls, Al-Azhar University, Cairo, Egypt.

*Corresponding author email: nahla.zohny@gmail.com 


\section{INTRODUCTION}

Edentulism is a growing problem, people loses their teeth for many reasons as tooth decay, periodontal diseases and some systemic diseases. The complete denture has been the most common treatment option for completely edentulous patients. For long time ago polymethyl methacrylate has been the preferred material for complete denture fabrication as it is a biocompatible material, insoluble in oral fluids and had reasonable cost. However, it had some limitations during conventional flasking and de-flasking laboratory steps. These laboratory procedures also increased processing errors and inaccuracies due to considerable human intervention $^{(1,2)}$.

These drawbacks have driven manufacturers to develop other alternative denture materials and new techniques for denture fabrication. Computer aided design and computer aided manufacturing (CAD/CAM) technologies have been introduced to dental field in the early 1980 's. The CAD/CAM technologies were based on digitalization of required data through the use of a scanner and software then fabrication of the desired restoration through a manufacturing machine. Two methods were used for fabrication of physical models in dentistry the first one is the subtractive method while the other is the additive method ${ }^{(3,4)}$.

In additive technique, the final desired shape is obtained by adding multiple layers of material on top of one another. The idea of this method is that the 3D-CAD model is sliced into many thin layers and the manufacturing machine uses this geometric data to build each layer sequentially until the part is completed. Therefore, it is called layered manufacturing (LM), direct digital manufacturing (DDM), three dimensional printing (3DP), solid freedom fabrication $(\mathrm{SFF})^{(5,6)}$

Denture retention is a functional process which depends on the salivary film thickness between fitting surface of complete denture and the underlying mucosa. The excellent adaptation of denture base and the border seal are the most important factors for achieving the best retention considered all proper conditions for salivary flow ${ }^{(7)}$.

\section{MATERIAL AND METHODS}

Eight completely edentulous patients were selected from the outpatient's clinic of removable prosthodontic department, faculty of dental medicine for girls, Al-Azhar University in-order to participate in this study. Patients were selected according to inclusion and exclusion criteria of the study. Patients were included when their age ranged from 40-55 years with class I angel's ridge relationship and can co-operate with a written questionnaire and the study protocol. Patients with any systemic or temporomandibular joint diseases or any ridge bony exostosis were excluded from the study. All research protocol was approved by the Research Ethics Committee (R.E.C) of the Faculty of Dental Medicine for Girls, Al- Azhar University. Patients medical and dental history were recorded in his file along with his clinical examination.

Maxillary and mandibular primary impressions were made in a properly selected stock trays using alginate impression materials. The secondary impressions were done using zinc oxide eugenol in the prefabricated acrylic resin special trays. The secondary impressions were then boxed and poured with type 3 dental stone to obtain the master casts. For each patient the casts were duplicated using addition curing duplicating silicone (REPLSIL 22 N) to obtain silicone model. The silicone models were then poured with type 3 dental stone to obtain the duplicate casts.

For the $\mathrm{CAD} / \mathrm{CAM} 3 \mathrm{D}$ printed maxillary denture, the original maxillary casts were sent to the lab for scanning using SHERA eco-scan 7 scanner and Dental wings software to scan the maxillary cast. Proper orientation of the cast should be obtained before scanning to ensure a proper scanning process. When scanning was completed, the 3D image of the scanned cast was displayed and stored with an extension of standard triangulation language (STL) files. (Fig. 1) 


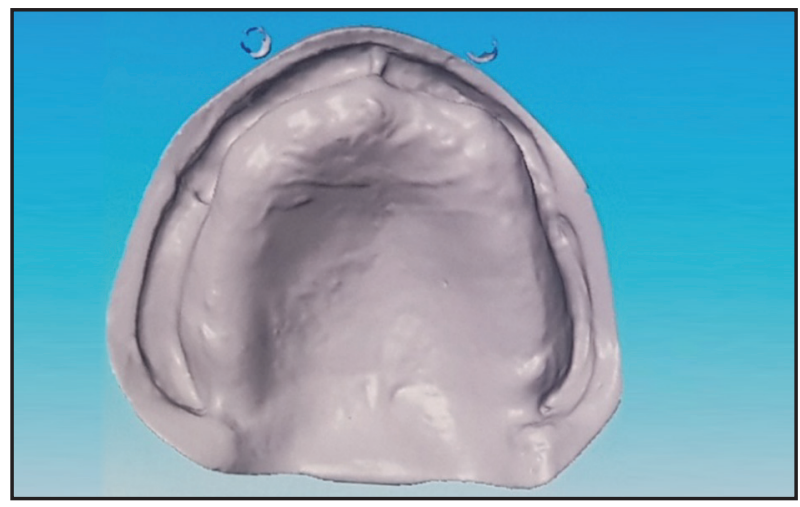

Figure (1) Digital image of the scanned cast.

The denture bases were printed according to Digital Light Projection (DLP) technology using Next-Dent Base liquid which is a monomer based on acrylic esters using Mogassam 3D printer. After the whole printing process was completed, the printed denture bases were rinsed in $99 \%$ isopropyl ethyl alcohol. After cleaning and drying of the printed denture bases. They were placed in an UV-light curing device for fifteen minutes for the final cure. The support structures were then removed, and the printed denture base were finished by fine bur and rotary tool then polished using Abraso-gum Acryl.

After the production of final 3D printed maxillary denture base, upper wax occlusal rims were made on the maxillary denture bases while the lower wax occlusal rims were fabricated over mandibular trial denture bases made of auto-polymerizing acrylic for recording jaw relations. The duplicated casts were used to fabricate the conventional dentures fabricated by the conventional compression flask technique. During delivery both dentures, the maxillary printed and conventional ones, were inserted inside patients' mouth for final adjustments checking for extensions, retention, stability and fine occlusal adjustments. (Fig. 2)

Each patient received only the first denture for one month then, a wash period of two weeks was performed. Then, the patient received the other denture and any further adjustment was carried out. Patients were classified into two groups, group (I)

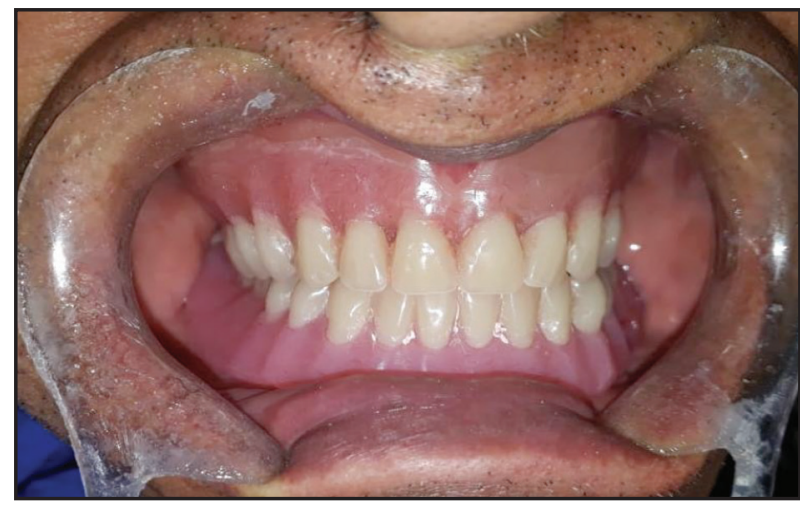

Figure (2) 3D printed upper denture with lower conventional denture after delivery.

received the conventional maxillary denture then their rapid prototyped denture while in Group II Patients received their rapid prototyped denture first then their conventional denture. Post insertion denture instructions were given to each patient.

Retention and patient satisfaction were assessed and recorded with each denture, for each patient at the day of delivery (baseline) and then after 1 month. Retention was measured for both groups using the universal testing machine. Modifications were made in each maxillary denture in-order to measure retention. The machine is connected to a computer and the value at which displacement took place was recorded and was reported as the retention value of the denture base. After recording five readings for each denture base their mean value was calculated and evaluated. Patient satisfaction was assessed for each patient through patient questionnaire and the visual analogue scale (VAS) at time of insertion and after 1 month.

All data recorded for each patient was tabulated and statistically analyzed.

\section{RESULTS}

Intra and intergroup comparisons were done utilizing paired t-test and independent t-test for parametric data, and Wilcoxon signed rank and Mann-Whitney $U$ tests for non-parametric data respectively. The significance level was set at $\mathrm{p} \leq$ 0.05 for all tests. 
Regarding retention, the results showed that there was no significant differences between both groups, group (I) who received the conventional denture first and group (II) who received the 3D printed denture (3DP) first $(\mathrm{p}=0.642)$ while in comparison of both types of dentures, there was a high significant difference between the conventional denture and the 3DP denture. the 3D printed denture $(22.92 \pm 2.64)$ had a significantly higher value than the conventional denture $(16.25 \pm 4.83)(\mathrm{p}=0.003)$.
Regarding patient satisfaction, that there was no significant differences between both groups, group (I) and group (II) including functional complaint about the denture, overall masticating ability, masticating ability for different types of food, effect on mental and daily life, overall denture satisfaction while in comparison of both types of dentures, there was a high significant difference between the conventional denture and the 3DP denture. The 3DP denture had better patient satisfaction than that of the conventional dentures.

Table (1) Mean, Standard deviation (SD) values of retention $(N)$ in different dentures.

\begin{tabular}{|c|c|c|c|c|}
\hline \multicolumn{5}{|c|}{ Retention } \\
\hline \multirow{2}{*}{ Groups } & \multirow{2}{*}{ Follow-up } & \multicolumn{2}{|c|}{ Denture } & \multirow{2}{*}{ p-value } \\
\hline & & Conventional & Printed & \\
\hline \multirow{3}{*}{ Group (I) } & Baseline & $17.33 \pm 6.66^{\mathrm{A}}$ & $19.67 \pm 2.08^{\mathrm{B}}$ & $0.669 \mathrm{~ns}$ \\
\hline & After 1 month & $20.00 \pm 5.29^{\mathrm{A}}$ & $23.33 \pm 1.53^{\mathrm{A}}$ & $0.405 \mathrm{~ns}$ \\
\hline & Overall & $18.67 \pm 5.57$ & $21.50 \pm 2.59$ & $0.317 \mathrm{~ns}$ \\
\hline \multirow{3}{*}{ Group (II) } & Baseline & $12.67 \pm 2.08^{\mathrm{A}}$ & $23.00 \pm 2.00^{\mathrm{A}}$ & $0.019^{*}$ \\
\hline & After 1 month & $15.00 \pm 2.65^{\mathrm{A}}$ & $25.67 \pm 0.58^{\mathrm{A}}$ & $0.012 *$ \\
\hline & Overall & $13.83 \pm 2.48$ & $24.33 \pm 1.97$ & $<0.001^{*}$ \\
\hline \multicolumn{2}{|c|}{ Overall } & $16.25 \pm 4.83$ & $22.92 \pm 2.64$ & $0.003^{*}$ \\
\hline
\end{tabular}

Different superscript letter indicate a statistically significant difference within the same vertical column and group *; significant $(p \leq 0.05)$ ns; non-significant $(p>0.05)$.

Table (2): Mean, Standard deviation (SD) values of overall denture satisfaction score in different dentures.

\begin{tabular}{ccccc}
\hline \multirow{2}{*}{ Groups } & \multicolumn{3}{c}{ Overall denture satisfaction } \\
& \multirow{2}{*}{ Follow-up } & \multicolumn{2}{c}{ Denture } & \multirow{2}{*}{ p-value } \\
\cline { 2 - 4 } & & Conventional & Printed & \\
\hline \multirow{3}{*}{ Group (I) } & Baseline & $3.33 \pm 0.58^{\mathrm{A}}$ & $3.33 \pm 0.58^{\mathrm{A}}$ & $1.000 \mathrm{~ns}$ \\
\cline { 2 - 4 } & After 1 month & $3.33 \pm 0.58^{\mathrm{A}}$ & $5.67 \pm 1.53^{\mathrm{A}}$ & $0.371 \mathrm{~ns}$ \\
\cline { 2 - 4 } & Overall & $3.33 \pm 0.52$ & $4.50 \pm 1.64$ & $0.317 \mathrm{~ns}$ \\
\hline \multirow{3}{*}{ Group (II) } & Baseline & $4.00 \pm 1.00^{\mathrm{A}}$ & $4.67 \pm 1.53^{\mathrm{A}}$ & $0.586 \mathrm{~ns}$ \\
\cline { 2 - 4 } & After 1 month & $3.00 \pm 0.00^{\mathrm{A}}$ & $4.00 \pm 1.00^{\mathrm{A}}$ & $0.371 \mathrm{~ns}$ \\
\cline { 2 - 4 } & Overall & $3.50 \pm 0.84$ & $4.33 \pm 1.21$ & $0.168 \mathrm{~ns}$ \\
\hline \multirow{2}{*}{} & Overall & $3.42 \pm 0.67$ & $4.42 \pm 1.38$ & $0.049^{*}$
\end{tabular}

Different superscript letter indicate a statistically significant difference within the same vertical column and group *; significant $(p \leq 0.05)$ ns; non-significant $(p>0.05)$ 


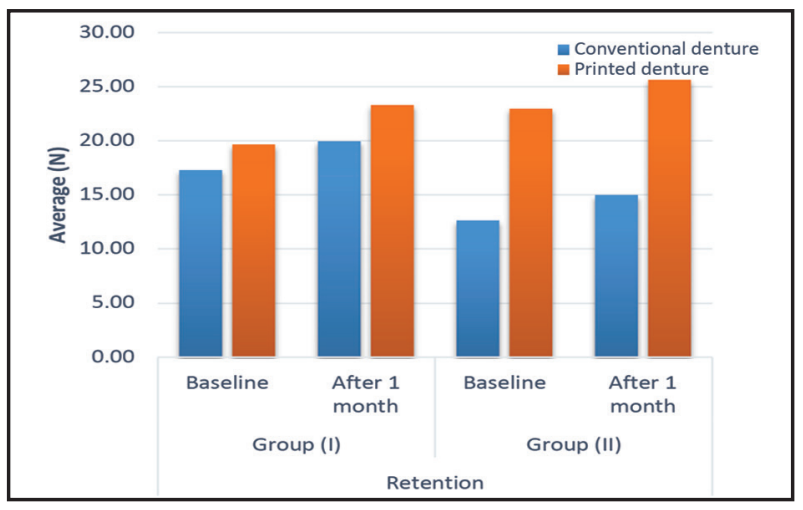

Figure (3) Bar chart showing average values of retention in different dentures.

\section{DISCUSSION}

For many decades, conventional complete denture has been the most applicable, gold standard method for treating edentulous patients restoring aesthetics, speech and masticatory function. Therefore, it has been chosen as a comparator in this study ${ }^{(8)}$.

Several studies had proven that the complete dentures fabricated from conventional techniques deform during processing. Linear distortion could be as much as $0.45 \%$ to $0.9 \%$ causing decreased adaptation of denture base to the mucosa. This leads to decrease in the retention, stability and support of the denture, the thing which has adverse consequences on the patient satisfaction and the clinician's chairside time required to perform the necessary adjustments. In an attempt to overcome these problems $\mathrm{CAD} / \mathrm{CAM}$ denture bases have been introduced. These bases were claimed to assume minimum fabrication distortion and consequentially better adaptation ${ }^{(9,10)}$. Hence, this study was performed to compare the retention and patient satisfaction of conventionally processed acrylic resin dentures versus CAD/CAM rapid prototyped dentures.

Regarding retention, when comparing retention values at insertion and after 1 month of both conventional and $\mathrm{CAD} / \mathrm{CAM} 3 \mathrm{D}$ printed denture, the results of this study showed that there was a significant difference between the conventional denture and the $3 \mathrm{D}$ printed denture. This indicated that the
3D printed denture had higher retention. The mean retention values after 1 month for digitally fabricated 3D printed dentures $(23.33 \mathrm{~N})$ recorded higher values than that of the conventionally fabricated dentures $(20.00 \mathrm{~N})$.

This may be supported by a study which found that the prosthesis which produced by $3 \mathrm{D}$ printing technology, adheres tightly to the tissue enhancing its retention, stability and transferring loads equally on the tissue ${ }^{(11)}$. Another study conducted that the 3D printing method had a higher technique accuracy than the conventional method ${ }^{(12)}$. Effective retention is attained by the close mucosal contact of the denture base ${ }^{(13)}$. Another study showed significantly higher scores for retention and stability for digitally fabricated dentures than the conventionally fabricated ones ${ }^{(4)}$. Another study found that $83 \%$ of the patients participated in the study had adequate retention for their digitally fabricated denture ${ }^{(14)}$.

On the other hand, the decreased retention values for conventional denture may be contributed to The higher dimensional changes in the conventionally heat cured acrylic resin dentures. This due to the release of residual internal stress along with polymerization shrinkage, thermal contraction during flask cooling and strain accompanying stress release during de-flasking ${ }^{(15)}$.

Regarding patient satisfaction, this study showed that there was no significant difference between patients who received the conventional denture first and those who received the 3D printed denture first. It also showed that there was a significant difference between the conventional denture and the 3D printed denture. This indicated that the 3D printed denture had higher patient's satisfaction regarding functional complaint of denture, overall masticatory ability, masticatory ability of different types of food and effect on mental and daily life. They also showed better overall satisfaction than the conventional dentures.

This may be consistent with a research which conducted that the surface quality of the $3 \mathrm{D}$ printed part showed high quality and the patient was satisfied 
during delivery ${ }^{(6)}$. A systematic review showed higher patient satisfaction and preference for the digital CDs where he stated that this may be due to the lack of blinding of patients which could have influenced their responses in favor of the new digital denture ${ }^{(16)}$.

On the other hand, another study showed that the $3 \mathrm{D}$ printed $\mathrm{CD}$ and the conventional ones were equally rated by the patients with respect to comfort and esthetics of denture, and overall satisfaction ${ }^{(12)}$. Another study showed that the ratings of the questionnaire of $\mathrm{CAD} / \mathrm{CAM}$ fabricated complete denture didn't differ significantly from those of the conventionally fabricated but overall, their satisfaction ratings of their digital CDs tended to be positive ${ }^{(14)}$.

\section{CONCLUSION}

Within the limitations of this study, it can be concluded that: there was no statistically significant difference between both groups, group (I) and group (II) regarding retention and patient satisfaction and that there was a statistically significant difference between the conventionally fabricated denture and the $\mathrm{CAD} / \mathrm{CAM} 3 \mathrm{D}$ printed denture regarding retention and patient satisfaction.

Dentures manufactured by $\mathrm{CAD} / \mathrm{CAM} 3 \mathrm{D}$ printing showed promising results yet, further studies are required with more sample size to evaluate the clinical outcomes. Continuous innovations in $\mathrm{CAD} /$ CAM dental technology enhanced the mechanical and clinical capability of dentures as it results in complete dentures with proper fit and best esthetics that meets with patient's requirements.

\section{REFERENCES}

1. Janeva N, Kovacevska G, Elencevski S, Panchevska S, Mijoska A, Lazarevska B. Advantages of CAD/CAM versus Conventional Complete Dentures - A Review. J Med Sci. 2018; 6:1498-502.

2. Masri G, Mortada R, Ounsi H, Alharbi N, Boulos P, Salameh Z.Adaptation of complete denture base fabricated by conventional, Milling, and 3-D printing techniques: An in vitro study. J Contemp Dent Pract.2020; 21: 367-71.

3. Bilgin MS, Baytaroglu EN, Erdem A, Dilber E. A review of computer-aided design/computer-aided manufacture techniques for removable denture fabrication. Eur J Dent. 2016; 10: 286-91.

4. Kattadiyil MT, Jekki R, Goodacre CJ, Baba NZ. Comparison of treatment outcomes in digital and conventional complete removable dental prosthesis fabrications in a predoctoral setting Presented at the Academy of Prosthodontics annual meeting, Kursaal, Berne, Switzerland. J Prosthet Dent. 2015; 114:818-25.

5. Torabi K, Farjood E, Hamedani S. Rapid Prototyping Technologies and their Applications in Prosthodontics, a Review of Literature. J Dent. 2015;16:1-9.

6. Ahmad M, Wahid M, Maidin N, Rahman M, Osman M, Elias I. A complete denture by rapid prototyping with reverse engineering approach. Int $\mathrm{J}$ Mech Mechatronics Eng. 2019;19:50-7.

7. Darvel BW, Clark RK. The physical mechanisms of complete denture retention. Br Dent J. 2000;189:248-52.

8. Singha S, Mishrab S, Chowdhary R. Patient expectations and satisfaction with conventional complete dentures: a systematic review. Tanta Dent J. 2019;16:55-67.

9. Goodacre BJ, Goodacre CJ, Baba NZ, Kattadiyil MT Comparison of denture base adaptation between CADCAM and conventional fabrication techniques. J Prosthet Dent. 2016;116:249-56.

10. Cristache C, Totu E, Iorgulescu G, Pantazi A, Dorobantu D, Nechifor A et al. Eighteen Months Follow-Up with Patient-Centered Outcomes Assessment of Complete Dentures Manufactured Using a Hybrid Nanocomposite and Additive CAD/CAM Protocol. J Clin Med. 2020; 9: 324-43.

11. Maryod WH. Retention of Removable Partial Denture Fabricated by Digital Designing and 3D Printing Technology - A Cross Over Study. Adv Dent Oral Heal. 2019;10:3-8

12. Inokoshi M, Kanazawa M, Minakuchi S. Evaluation of a complete denture trial method applying rapid prototyping. Dent Mater J. 2012;31:40-6

13. Kumar VH, Surapaneni H, Ravikiran V, Chandra BS, Balusu S, Reddy VN. Retention of denture bases fabricated by three different processing techniques - An in vivo study. J Int Soc Prev Community Dent. 2016;6:245-50.

14. Saponaro PC, Yilmaz B, Johnston W, Heshmati RH, McGlumphy EA. Evaluation of patient experience and satisfaction with CAD-CAM-fabricated complete dentures: A retrospective survey study. J Prosthet Dent. 2016;116:524-8.

15. Aboelroos E, Rady A. The Effect of Two Packing Techniques on Adaptation of Resin Denture Base Materials. Egypt Dent J. 2016;62:1169-76.

16. Wimmer T, Gallus K, Eichberger M, Stawarczyk B. Complete denture fabrication supported by CAD/CAM. J Prosthet Dent. 2016;115:541-6. 